\title{
Programa Exploratório de Ensaios de Proficiência em Medidas de Emissão Radiada
}

\author{
Jorge Vallim Guimarães, Maurício Henrique Costa Dias e José Carlos Araujo dos Santos ${ }^{1}$
}

\begin{abstract}
Resumo-Ensaios de proficiência são um método de grande relevância em processos de acreditação de laboratórios no contexto de metrologia. Adicionalmente, o crescente mercado de produtos de tecnologia da informação e de telecomunicações impõe a necessidade de testes de conformidade com normas de compatibilidade eletromagnética (CEM). $O$ presente trabalho relata um programa de ensaios de proficiência recém-concluído envolvendo sete laboratórios brasileiros capacitados a realizar medidas em CEM. Mais especificamente, o objetivo foi avaliar o desempenho dos laboratórios em medições de emissão radiada. O resultado global foi satisfatório, mas a análise, ainda que exploratória, apontou a necessidade de ações preventivas e corretivas para alguns participantes.
\end{abstract}

Palavras-Chave-Ensaios de proficiência, comparação interlaboratorial, compatibilidade eletromagnética, emissão radiada.

\begin{abstract}
In metrology, proficiency testing plays a major role in accreditation processes of laboratories. Moreover, the growing market for information technology and telecommunications equipments demands the need for electromagnetic compatibility (EMC) compliance tests. The present work summarizes the main achievements of a recently concluded proficiency testing scheme carried out with seven EMC Brazilian laboratories. Assessing the laboratories performances in radiated emission measurements was the main goal of that testing. The overall result was fine, though the analysis has pointed out the need for preventive and corrective actions for some participants.
\end{abstract}

Index Terms-Proficiency testing, interlaboratory comparison, electromagnetic compatibility, radiated emission.

\section{INTRODUÇÃO}

No contexto industrial eletro-eletrônico, um tema de grande importância é a compatibilidade eletromagnética (CEM). Todo equipamento eletro-eletrônico é uma fonte em potencial de distúrbios eletromagnéticos, que podem gerar interferência eletromagnética (IEM) em dispositivos ou sistemas ao redor, comprometendo os desempenhos esperados de todos. Ou seja, equipamentos eletro-eletrônicos geram e estão sujeitos a IEM. Em essência, a compatibilidade eletromagnética pode ser definida como a harmonia de uso

Seção de Engenharia Elétrica, Instituto Militar de Engenharia, Rio de Janeiro, Brasil. E-mails: jorge@vallim.eng.br; mhcdias@ime.eb.br; e araujo@ime.eb.br. desses equipamentos em um mesmo ambiente, sem prejuízo das funções originais de cada um [1]-[2].

Grande parte dos cuidados necessários e indicados para se obter a CEM está registrada em normas e recomendações emitidas por organismos internacionais reconhecidos, como o IEC (International Electrotechnical Comitte) e o IEEE (Institute of Electrical and Electronic Engineering). Entretanto, não compete a tais instituições impor suas normas a ninguém. A adoção dessas normas se dá por opção dos interessados e/ou por leis e regulamentos de cada país. No primeiro caso, o interesse é normalmente comercial, buscando atender a requisitos exigidos por potenciais clientes no mercado externo. Para assegurar a qualidade e a segurança para os consumidores do mercado interno, agências reguladoras de cada país se encarregam de cobrar o atendimento a normas de CEM. No Brasil, a ANATEL e a ANVISA são os principais agentes nesse contexto.

Seja para obter liberação de comercialização por parte de alguma agência reguladora, seja para atender a demandas de clientes, os equipamentos precisam passar por testes de conformidade em CEM. Em ambos os casos, apenas laudos emitidos por laboratórios acreditados costumam ter respaldo suficiente. No Brasil, o órgão responsável pela acreditação de laboratórios de ensaio e calibração é o INMETRO (Instituto Nacional de Metrologia), e a norma principal de referência adotada é a NBR ISO/IEC 17025 [3].

Em processos de acreditação (ou de sua manutenção), um dos mecanismos de controle da qualidade de laboratórios é a participação em ensaios de proficiência [3]. Os requisitos mínimos para um laboratório se integrar a este tipo de iniciativa são estabelecidos em [4]. Uma das formas mais aceitas de realização de ensaios de proficiência é por comparação interlaboratorial. O INMETRO adota como norma-base sobre este assunto o Guia 43-1 da ABNT (Associação Brasileira de Normas Técnicas), que trata do planejamento, das condições de realização e da avaliação de resultados de um programa de ensaios naqueles moldes [5].

O INMETRO mantém 13 Comissões Técnicas (CT) de apoio à atividade de acreditação de laboratórios. A comissão que trata especificamente da área eletro-eletrônica é a CT-08. Uma das atividades recentes estimuladas por esta comissão foi um programa exploratório de ensaios por comparação interlaboratorial de medidas em CEM. Mais especificamente, o CT-08 adotou como referência para a condução dos ensaios a norma IEC/CISPR 22 [6], que trata da CEM de equipamentos de tecnologia da informação (TI), e especifica testes de emissão conduzida e radiada. $\mathrm{O}$ primeiro autor do presente texto atuou diretamente na coordenação daquele 
programa, que foi dividido em duas partes. Na primeira, medidas de emissão conduzida de uma fonte chaveada foram realizadas por seis laboratórios brasileiros e um argentino, e os resultados principais foram resumidos em [7] e [8]. A segunda etapa foi realizada em 2007, e contou com a participação de sete laboratórios brasileiros em medidas de emissão radiada de dispositivos geradores de ruído. A descrição desta etapa de ensaios em emissão radiada é o foco deste artigo.

Cumpre destacar ainda algumas importantes iniciativas de outros autores correlatas ao presente trabalho. Em [9] e [10], os autores realizaram ensaios de proficiência por comparação interlaboratorial de medições nos moldes descritos em [5]. Seis laboratórios participaram dos ensaios, cujo corpo de prova padrão foi um filtro de linha, e a grandeza medida foi a atenuação de saída. Ou seja, embora dentro do contexto eletro-eletrônico, aqueles trabalhos não estavam relacionados diretamente a CEM. Mais recentemente, foi relatada a realização de ensaios de proficiência em diversas frentes da CEM (emissão radiada e conduzida, imunidade a IEM radiada e conduzida, imunidade a descargas eletrostáticas, entre outras) [11]-[12]. Entretanto, a comparação se limitou a dois laboratórios. Deve-se destacar que, em ambos os trabalhos, aqueles autores também analisaram o ponto de vista dos laboratórios, em particular quanto a metodologias de determinação da incerteza de cada ensaio. Estas incertezas são dados fundamentais que os laboratórios precisam fornecer ao coordenador de um programa de ensaios para realização da análise estatística global dos resultados. Por fim, cumpre acrescentar que há iniciativas similares à do presente trabalho em outros países, ainda que relativamente recentes, como em [13]-[14].

Este artigo foi estruturado como segue. A seção II resume as características principais de ensaios de proficiência por comparações interlaboratoriais. O programa de ensaios em emissão radiada realizado é descrito na seção seguinte. $\mathrm{Na}$ seção IV a análise dos resultados é apresentada. O presente texto é concluído na seção seguinte.

\section{ENSAIOS DE PROFICIÊNCIA POR COMPARAÇÕES INTERLABORATORIAIS}

\section{A. Planejamento de Ensaios}

São atribuições do INMETRO o planejamento e a execução das atividades de acreditação de laboratórios e de organismos de certificação. O processo de acreditação tem como objetivo atestar que o laboratório possui competência técnica para a realização de ensaios ou calibrações.

Ensaios de proficiência são parte integrante de um processo de acreditação. A demonstração da proficiência se dá pela comparação de resultados do laboratório com padrões conhecidos ou com resultados de outros laboratórios. A condução de ensaios de proficiência é orientada pelo Guia 43-1 [5], que estabelece seis formas distintas: comparações de medições; ensaios interlaboratoriais; ensaios de partidas de amostras; ensaios qualitativos; ensaios de valores conhecidos; e programa de processo-parcial. Todas estas formas de ensaio envolvem, necessariamente, comparações interlaboratoriais, à exceção das duas últimas, que podem ser executadas por um único laboratório.

Um programa de ensaios de proficiência deve ser conduzido por um coordenador (pessoa ou instituição) com uma equipe que reúna os seguintes tipos de conhecimentos: técnicos, na área objeto do ensaio; estatísticos; e de metrologia. Via de regra, a coordenação envolve atividades de planejamento, condução e acompanhamento do programa, além de análise dos resultados, na fase final.

No planejamento de um programa de ensaios, o coordenador deve preparar um documento de orientação geral aos laboratórios participantes. As principais informações que devem constar no texto diretor são as seguintes [5]: organização que conduz o programa; critérios para participação no programa; natureza e propósito do programa; laboratórios envolvidos; itens de ensaio selecionados; logística de preparação e execução dos ensaios; métodos de ensaios; técnicas de avaliação de desempenho; metodologias de análise dos resultados; instruções para preparação da documentação. Além desses dados, o coordenador pode solicitar outros detalhes julgados relevantes para a correta análise comparativa de todos os resultados.

O relatório final do programa também deve seguir um padrão mínimo [5]. Deve conter a descrição clara do item de ensaio utilizado, incluindo detalhes da preparação, e estudos de estabilidade e homogeneidade realizados. O procedimento adotado para determinação de valores de referência também deve ser descrito, e quando for o caso, evidências de rastreabilidade e incerteza de qualquer valor de referência devem ser citadas. Ainda, o método utilizado para análise estatística dos resultados globais deve ser indicado, seguido da análise propriamente dita.

Todo programa de ensaios deve seguir alguns preceitos de confiabilidade e ética [5]. Em especial, a identidade dos laboratórios envolvidos deve ser de conhecimento do menor número de pessoas possível, além do coordenador. Se houver a necessidade de divulgação de dados que revelem a identidade de algum laboratório, isto deve ser previamente acertado entre as partes envolvidas. Laboratórios podem renunciar à confidencialidade para discussão e assistência técnica sobre seu desempenho no programa.

\section{B. Métodos Estatísticos para Análise de Ensaios}

O Guia 43-1 recomenda que a análise de resultados de um programa de ensaios de proficiência compreenda algumas etapas fundamentais: verificação da homogeneidade e estabilidade dos mensurandos (itens de ensaio); determinação do valor verdadeiro de consenso designado (VVC); e análise de desempenho [5]. Metodologias para realização dessas etapas são descritas resumidamente em [5], mas o coordenador tem alguma flexibilidade na escolha dos métodos de análise, conquanto que preceitos comuns de estatística sejam respeitados, e que haja consenso entre os participantes.

A ISO (International Organization for Standardization) lançou uma norma em 2005 - ISO 13528 [15], com o objetivo de complementar o Guia 43-1 na escolha dos métodos estatísticos de análise. Esta norma indica um 
fluxograma com todas as ações a serem tomadas nesse contexto, para os diferentes tipos de ensaios de proficiência. A ação inicial envolve a verificação de homogeneidade e estabilidade, para a qual a norma descreve um método específico [15]. Em seguida, é preciso verificar se o VVC e sua incerteza devem ser estabelecidos antes ou depois dos ensaios. Em ambos os casos, ao final do programa deve ser realizada a análise dos resultados.

Há cinco alternativas previstas para determinação do valor designado ou valor verdadeiro convencional [5]. A escolha depende da disponibilidade de valores que podem ser: conhecidos; de referência certificados; de referência; de consenso de laboratórios especialistas; e de consenso de laboratórios participantes. Esta última opção é bastante utilizada, principalmente em ensaios inéditos ou em ensaios exploratórios. Neste caso, o VVC escolhido é normalmente um momento estatístico representativo, como a média ou a mediana dos valores de todos os laboratórios, por exemplo.

As principais formas de quantificação do desempenho são apresentadas no Guia 43-1 [5], e também na norma complementar ISO 13528 [15]. Pode ser a diferença (bias) entre o $\operatorname{VVC}(X)$ e o resultado do laboratório $(x)$, ou a diferença percentual $(\Delta)$ :

$$
\Delta=100(x-X) / X
$$

Há ainda o z-score $(z)$ e o erro normalizado $\left(E_{n}\right)$, dados respectivamente por:

$$
\begin{gathered}
z=(x-X) / \sigma \\
E_{n}=(x-X) / \sqrt{U_{l a b}^{2}+U_{r e f}^{2}}
\end{gathered}
$$

onde $\sigma$ representa o desvio-padrão estabelecido para os ensaios, e $U_{l a b}$ e $U_{r e f}$ as incertezas expandidas associadas ao laboratório e ao item de ensaio, respectivamente. $\mathrm{O}$ documento principal de orientação para cálculo de incertezas em procedimentos metrológicos é o GUM [16], para o qual o INMETRO possui versão traduzida e atualizada [17].

Uma contribuição importante de [15] é a indicação de um algoritmo robusto de estimação do VVC e do desvio-padrão dos ensaios, quando estes valores são definidos a partir das medições dos laboratórios envolvidos. Outras opções de determinação do valor designado muito usadas na prática pelo pessoal de metrologia envolvem a busca e eliminação de valores dispersos (outliers), como nos métodos de Grubbs [18] e Dixon [19], por exemplo, seguidas de análise estatística convencional para o cálculo de $X$ e $\sigma$. A indicação explícita de um método em uma norma representa um consenso entre especialistas no assunto. Neste sentido, é provável que o método robusto venha a se tornar um padrão de escolha por parte de coordenadores de ensaios.

A avaliação do desempenho pode se dar por um consenso de especialistas acerca da adequação técnica dos resultados, ou pela definição de uma probabilidade de abrangência para aceitação dos resultados (normalmente 95\% ou 99\%). Esta última opção é comumente realizada a partir dos parâmetros $z$ e $E_{n}$. No primeiro caso, resultados dentro de dois desviospadrão $(|z| \leq 2)$ são considerados aceitáveis. A reprovação ocorre quando $|z|>3$ e resultados intermediários são tratados como questionáveis. Ao analisar o erro normalizado, os resultados são aceitáveis quando $\left|E_{n}\right| \leq 1$ (não há faixa intermediária de qualificação, como no $z$-score). Resultados questionáveis e inaceitáveis são um indicador da necessidade de ações preventivas e corretivas, respectivamente, que um laboratório deve tomar quanto a seus procedimentos de medições.

\section{PRograma de ENSAIOS EM EMISSÃo RAdiAdA}

Um programa inédito de ensaios de proficiência em medidas de compatibilidade eletromagnética foi montado, por estímulo da CT-08 do INMETRO e por desejo de laboratórios (em sua maioria) brasileiros envolvidos com CEM. A opção mais pertinente para o programa foi o método de comparação de medições. O programa previa a realização de rodadas exploratórias de ensaios em emissão conduzida e radiada.

Seguindo as orientações da CT-08, adotou-se a CISPR 22 [6] como referência adicional aos documentos que normatizam ensaios de proficiência. Os principais resultados dos ensaios com emissão conduzida foram apresentados em [7]-[8]; a descrição da rodada de ensaios em emissão radiada, que ocorreu na seqüência, ao longo de 2007 é apresentada no presente artigo. Nestes ensaios, seis laboratórios brasileiros acreditados em medições de CEM participaram: CIENTEC; CPqD; IPE; INPE; NMI; GEMCO-UFSC. Além desses, o laboratório de CEM do Centro Tecnológico do Exército Brasileiro (CTEx) também se integrou ao programa.

$\mathrm{O}$ coordenador do programa não interferiu nas metodologias de ensaio utilizadas pelos laboratórios. Todo aparato de teste envolvido (antenas, receptores, ambiente de ensaio, etc.), bem como métodos de calibração, de medição e de determinação de incertezas eram de responsabilidade e competência dos laboratórios, cada qual com suas peculiaridades. Quanto ao atendimento à [6], o limite superior freqüencial adotado foi de $1 \mathrm{GHz}$, já que a acreditação dos laboratórios participantes contemplava apenas até aquele limite. A versão atual da norma subiu para $6 \mathrm{GHz}$ a freqüência máxima de testes.

Duas fontes geradoras de ruído foram escolhidas como dispositivos de testes: uma fonte CNE III da York; e uma fonte desenvolvida e montada pelo próprio coordenador (Fig. 1). A primeira apresenta um espectro relativamente contínuo, que dificulta a análise de resultados, do ponto de vista de ensaios de proficiência. A fonte montada, por outro lado, apresenta um espectro de picos isolados, similar ao de um filtro comb, que facilita a comparação de resultados, por conter um número limitado de harmônicas para se analisar. A Fig. 2 mostra o espectro desta fonte, gerado por um dos participantes do ensaio. Cumpre destacar que os argumentos que justificam a opção por este tipo de fonte para ensaios de proficiência em emissão radiada também foram abordados em [14], corroborando a presente escolha. 

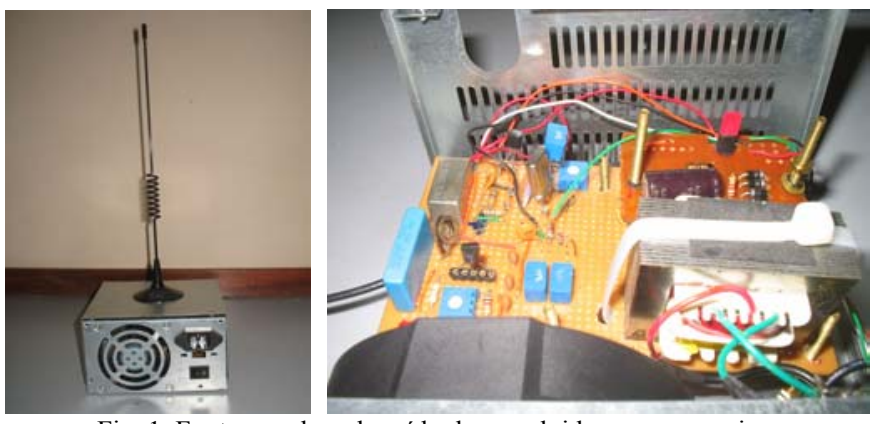

Fig. 1. Fonte geradora de ruído desenvolvida para os ensaios.

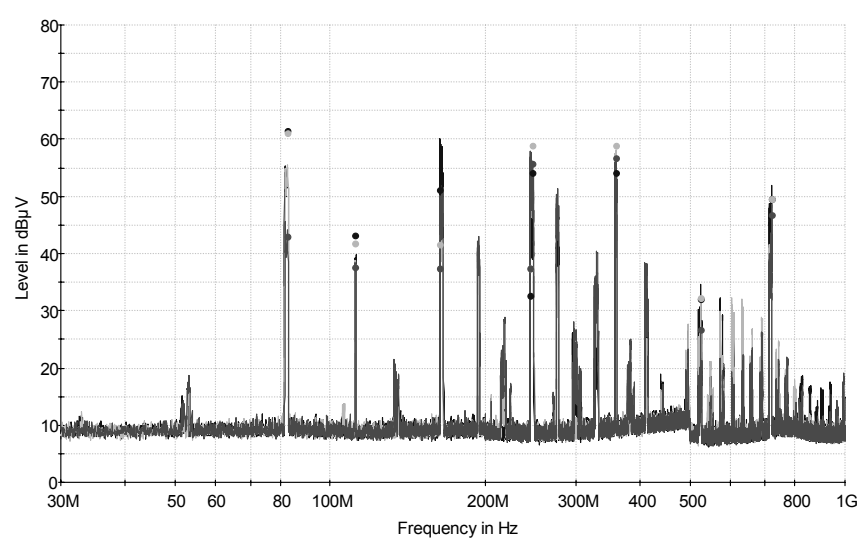

Fig. 2. Espectro de emissão da fonte montada, medido por um dos laboratórios.

Foi acordado entre todos os laboratórios participantes que as medidas seriam de quase-pico, como previsto em [6]. Medições em polarização vertical e horizontal foram feitas. No programa em questão, um aspecto importante a destacar foi a heterogeneidade dos ambientes de teste. Seis laboratórios possuíam câmaras anecóicas ou semi-anecóicas, como no exemplo da Fig. 3, algumas com dimensões que permitiam medidas até $10 \mathrm{~m}$, outras apenas até $3 \mathrm{~m}$. Um dos laboratórios possuía uma célula GTEM. Para padronizar a análise, assumiu-se que o ambiente de referência era a câmara (anecóica ou semi), com distância de medição a $3 \mathrm{~m}$. Para os resultados medidos a $10 \mathrm{~m}$ ou na célula GTEM, adotou-se um fator de correção de $10,5 \mathrm{~dB}$, como sugerido em [6], que corresponde à perda de espaço livre de 3 para $10 \mathrm{~m}$ $\left(20 \cdot \log _{10} 10 / 3\right)$.

\section{AnÁlise dos Resultados}

Neste artigo, a análise dos resultados fornecidos pelos laboratórios assume a metodologia robusta recomendada em [15]. O desempenho é avaliado com base nos parâmetros $z$ e $E_{n}$. Para o cálculo do erro normalizado, é preciso estimar a incerteza expandida do VVC. Esta atribuição é do coordenador do programa, apesar de se referir a um cálculo de incerteza, pois o VVC foi assumido como a média dos valores medidos em todos os laboratórios participantes. Há várias metodologias para se estimar esta incerteza, como por exemplo, usar um valor de referência para o tipo de ensaio realizado. Uma boa indicação para o tipo de ensaios deste programa foi encontrada na CISPR 16-4-1 [20], que recomenda o limite de $5,2 \mathrm{~dB}$ para a incerteza expandida $U_{r e f}$. Entretanto, como se assumiu o método robusto da ISO 13528, neste artigo a incerteza expandida foi calculada de acordo com o recomendado naquela norma [15], ou seja:

$$
U_{r e f}=k \cdot 1,25 \cdot \sigma / \sqrt{p}
$$

onde $k$ é o fator de abrangência (normalmente igual a 2, como neste trabalho), definido em [16], $\sigma$ é o desvio-padrão obtido pelo método robusto, e $p$ o número de laboratórios considerados (sete no caso deste programa).

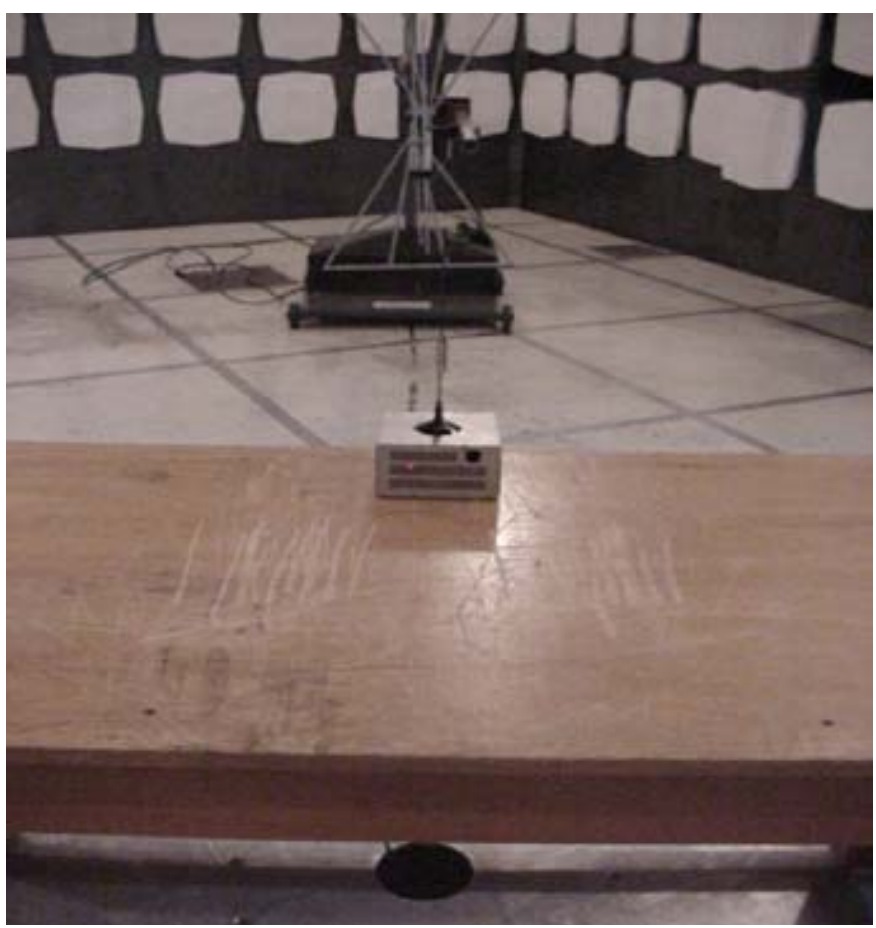

Fig. 3. Configuração de teste na câmara semi-anecóica de um dos laboratórios.

Como mencionado na seção anterior, a fonte de testes utilizada apresentava um espectro raiado, como o da Fig. 2. Para a análise de desempenho, foram escolhidas as cinco freqüências em que o campo medido, em polarização vertical ou horizontal, era mais intenso: $82 ; 165 ; 246 ; 359$; e 717 $\mathrm{MHz}$. Os valores máximos reportados pelos laboratórios nestas cinco freqüências estão representados na Fig. 4. A Tabela I apresenta os momentos estatísticos (médias, desviospadrão e incertezas de referência) calculados para cada freqüência, segundo o método robusto indicado em [15]. Tendo determinado $X$ e $\sigma$, calculou-se o $z$-score para todos os laboratórios, a partir de (2). Os valores obtidos estão representados na Fig. 5. $E_{n}$ foi calculado como em (3) assumindo-se as incertezas reportadas por cada laboratório e as incertezas de referência em cada freqüência calculadas por (4). A Fig. 6 apresenta os valores de $E_{n}$ assim determinados.

Por questões de sigilo das informações, conforme preconiza [5], os laboratórios não podem ser identificados na análise aqui apresentada. De fato, os dados aqui listados são um resumo da análise mais detalhada encontrada em [21], que também preserva o sigilo já citado, mas considera o desempenho individual de cada laboratório, correlacionando os resultados de todas as freqüências com potenciais falhas 
nos procedimentos de medição e/ou na estimação das incertezas, entre outros aspectos observados pelo coordenador do programa.

\section{Campo Elétrico Medido}

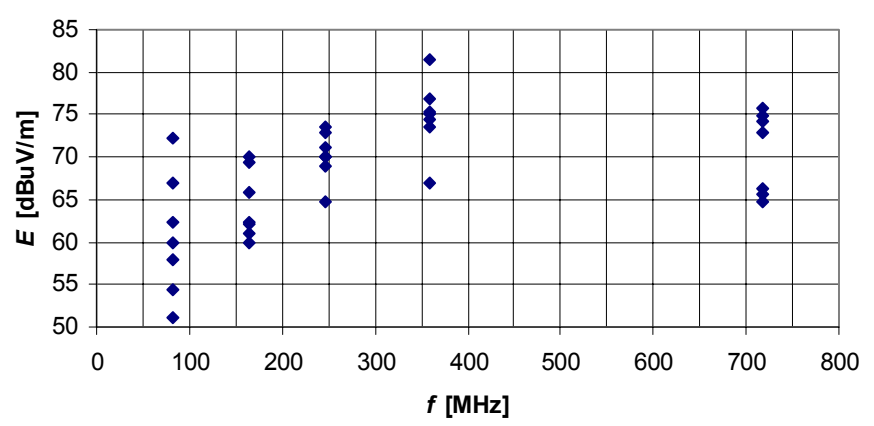

Fig. 4. Valores de campo radiado medidos nas cinco freqüências escolhidas.

TABELA I

Momentos Estatísticos Robustos OBtidos

\begin{tabular}{|c|c|c|c|}
\hline Freqüência $[\mathrm{MHz}]$ & $X[\mathrm{~dB} \mu \mathrm{V} / \mathrm{m}]$ & $\sigma[\mathrm{dB}]$ & $U_{\text {ref }}[\mathrm{dB}]$ \\
\hline 82 & 60,61 & 8,04 & 7,60 \\
165 & 63,88 & 3,77 & 3,56 \\
246 & 70,41 & 2,20 & 2,08 \\
359 & 75,01 & 2,36 & 2,23 \\
717 & 71,10 & 4,76 & 4,50 \\
\hline
\end{tabular}

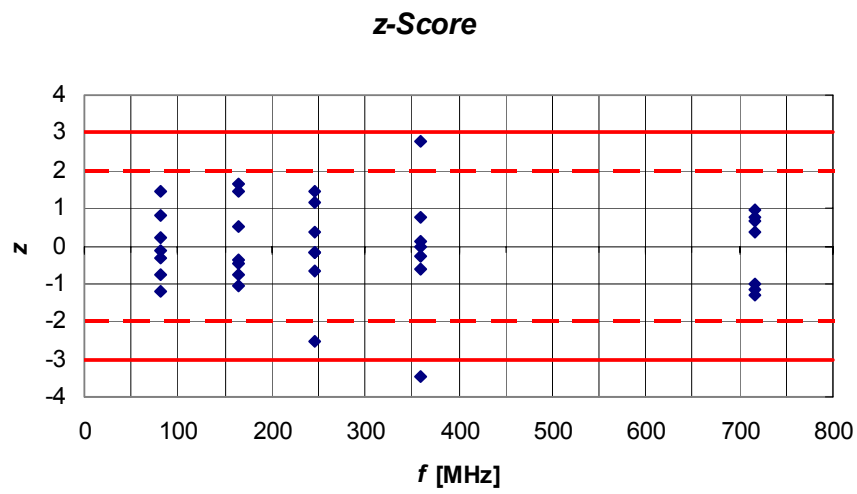

Fig. 5. Valores de $z$-score calculados.

\section{Erro Normalizado}

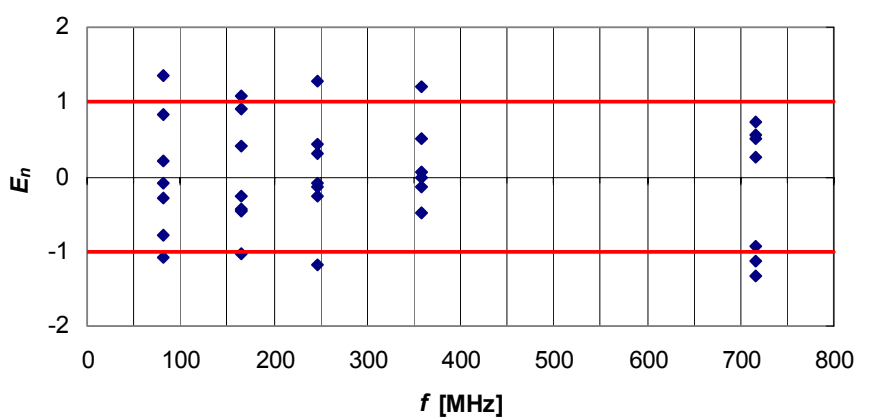

Fig. 6. Erros normalizados calculados.

$\mathrm{Na}$ análise por z-score, a maioria dos resultados foi considerada satisfatória. De um total de 35, apenas dois valores caíram na faixa de ações preventivas $(2<|z| \leq 3)$, e um único na de ações corretivas $(|z|>3)$. Pode-se afirmar que o resultado global por este indicador, portanto, foi bom.

$\mathrm{O}$ erro normalizado, por sua vez, indicou resultados mais pessimistas, com oito dos 35 valores na faixa insatisfatória $\left(\left|E_{n}\right|>1\right)$. A explicação para a discrepância com o z-score está no fato que o cálculo do erro normalizado é afetado diretamente pela variação no valor das incertezas de medição reportadas pelos laboratórios [9]. Os valores de $U_{l a b}$ informados, detalhados em [21], variaram de tal forma que sugerem a necessidade de harmonização do uso das normas de referência, [16], [17] e [20], em futuros ensaios.

\section{COMENTÁRIOS FINAIS}

Este trabalho descreveu um programa de ensaios de proficiência por comparação interlaboratorial em medidas de compatibilidade eletromagnética recém-realizado envolvendo sete laboratórios brasileiros. $\mathrm{O}$ foco dos ensaios foi a medição de emissão radiada de equipamentos de TI e telecomunicações, normatizada pela CISPR 22 [6].

As principais orientações da norma-base para ensaios de proficiência, o Guia 43-1 [5], foram sintetizadas neste artigo. Algumas considerações sobre métodos estatísticos de análise foram discutidas, em especial o papel da norma ISO 13528 [15] neste contexto. O programa de ensaios realizado foi descrito resumidamente, indicando aspectos técnicos relevantes como o dispositivo de teste adotado (fonte geradora de ruído impulsivo) e os ambientes de ensaios.

Os resultados fornecidos pelos laboratórios foram analisados através dos parâmetros $z$-score e erro normalizado, seguindo as orientações de [15] para os cálculos. Da análise do primeiro, o desempenho global dos laboratórios foi bom, já que houve apenas um valor de $z$ insatisfatório de um total de 35. Entretanto, a análise por erro normalizado apresentou mais valores fora da faixa aceitável, indicando potenciais problemas ou erros na estimação das incertezas de medição por parte de alguns dos laboratórios participantes.

Deve-se destacar que a rodada exploratória aqui relatada resumidamente representou uma primeira iniciativa de ensaios de proficiência envolvendo quase todos os laboratórios brasileiros de medidas de CEM. Outras iniciativas nesse mesmo contexto têm acontecido nos últimos anos, e outras ainda virão. O próprio INMETRO, através de sua CT-08 já vem planejando novas rodadas de ensaios, a participação de mais laboratórios (inclusive internacionais), e também a inclusão de outros testes em CEM, como em [11].

\section{AGRADECIMENTOS}

Os autores agradecem aos sete laboratórios participantes, e em especial aos técnicos e engenheiros que participaram direta ou indiretamente dos ensaios.

\section{REFERÊNCIAS}

[1] C. R. Paul, Introduction to Electromagnetic Compatibility, $2^{\text {nd }} \mathrm{Ed}$, Wiley, 2006.

[2] M. I. Montrose, E. M. Nakauchi, Testing for EMC Compliance: Approaches and Techniques, Wiley, 2004.

[3] Requisitos Gerais para a Competência de Laboratórios de Ensaio e Calibração, Norma NBR ISO/IEC 17025, Associação Brasileira de Normas Técnicas, 2005. 
[4] Requisitos sobre a Participação dos Laboratórios de Ensaio e de Calibração em Atividades de Ensaio de Proficiência, Norma NITDICLA-026, INMETRO, 2005.

[5] Ensaios de Proficiência por Comparações Interlaboratoriais - Parte 1: Desenvolvimento e Operação de Programas de Ensaios de Proficiência, Norma ABNT ISO/IEC Guia 43-1, Associação Brasileira de Normas Técnicas, 1999.

[6] Information technology equipment - Radio disturbance characteristic Limits and methods of measurement, Norma CISPR 22, International Eletrotechnical Comission, 2005.

[7] J. V. Guimarães, J. C. A. Santos, C. P. Saraiva, "Ensaio de Proficiência por Comparação de Medições do Ensaio de Emissão Conduzida segundo a CISPR 22", in ENQUALAB 2007 - Congresso da Qualidade em Metrologia, São Paulo - SP, Jun 2007.

[8] J. V. Guimarães, M. H. C. Dias, J. C. A. Santos, "Ensaios de Proficiência por Comparação de Medições para Emissões Conduzidas e Radiadas", in $7^{\circ}$ Seminário Internacional de Metrologia Elétrica (VII SEMETRO), Belo Horizonte - MG, Set 2007.

[9] K. T. Soletto, Comparações Interlaboratoriais para Laboratórios de Compatibilidade Eletromagnética, tese de doutorado, Universidade de São Paulo, São Paulo - SP, 2004.

[10] K. T. Soletto, J. J. A. Ferreira, "Comparações Interlaboratoriais para Laboratórios de Compatibilidade Eletromagnética", in ENQUALAB 2004 - Congresso da Qualidade em Metrologia, São Paulo - SP, Jun 2004.

[11] F. Nyland, Contribuição ao Ensaio de Proficiência em Compatibilidade Eletromagnética, dissertação de mestrado, Universidade Federal de Santa Catarina, Florianópolis - SC, Dez 2007.

[12] A. Raizer, F. Nyland, M. H. Amaral, "Cálculo de incerteza para compatibilidade eletromagnética", in $7^{\circ}$ Seminário Internacional de Metrologia Elétrica (VII SEMETRO), Belo Horizonte - MG, Set 2007.
[13] B. S. Rao, K. Varadan, "EMI proficiency testing program - conducted emission measurement", in 2004 IEEE International Symposium on Electromagnetic Compatibility, Piscataway - EUA, vol. 3, pp. 925-930, Ago 2004.

[14] K. Osabe, R. Watanabe, A. Maeda, M. Yamaguchi, "Inter-laboratory Comparison Result as the Proficiency Testing Program of EMI Test Sites in Japan" in 2007 IEEE International Symposium on Electromagnetic Compatibility, Honolulu - EUA, vol. 1, pp. 1-6, Jul 2007.

[15] Statistical methods for use in proficiency testing by interlaboratory comparisons, Norma ISO 13528, International Organization for Standardization, 2005.

[16] Guide to the expression of uncertainty in measurement (GUM), Norma ISO/IEC Guide 98, International Organization for Standardization, 1995.

[17] Expressão da Incerteza de Medição na Calibração, Norma NITDICLA 21-03, INMETRO, 2007.

[18] F. E. Grubbs, "Sample Criteria for Testing Outlying Observations", Annals of Mathematical Statistics, vol. 21, no. 1, pp. 27-58, 1950.

[19] W. J. Dixon, "Analysis of Extreme Values", Annals of Mathematical Statistics, vol. 21, no. 4, pp. 488-506, 1950.

[20] Specification for radio disturbance and immunity measuring apparatus and methods - Part 4-1: Uncertainties, statistics and limit modeling Uncertainties in standardized EMC tests, Norma CISPR 16-4-1, International Eletrotechnical Comission, 2005.

[21] J. V. Guimarães, Ensaios de Proficiência em Compatibilidade Eletromagnética: Programa Exploratório de Medidas de Emissão Radiada, dissertação de mestrado, Instituto Militar de Engenharia, Rio de Janeiro, Mai 2008. 